\title{
Article
}

\section{Differential microRNA Expression in USP8-Mutated and Wild-Type Corticotroph Pituitary Tumors Reflect the Difference in Protein Ubiquitination Processes}

\author{
Mateusz Bujko ${ }^{1}$, Paulina Kober ${ }^{1}$, Joanna Boresowicz ${ }^{1}$, Natalia Rusetska ${ }^{1}$, Natalia Zeber-Lubecka ${ }^{2}$, \\ Agnieszka Paziewska ${ }^{3}$, Monika Pekul ${ }^{4}$, Grzegorz Zielinski ${ }^{5}$, Andrzej Styk ${ }^{5}$, Jacek Kunicki ${ }^{6}$, Jerzy Ostrowski ${ }^{2,7}$, \\ Janusz A Siedlecki ${ }^{1}$ and Maria Maksymowicz ${ }^{4, *}$
}

Citation: Bujko, M.; Kober, P.; Boresowicz, J.; Rusetska, N.; Zeber-Lubecka, N.; Paziewska, A.; Pekul, M.; Zielinski, G.; Styk, A.; Kunicki, J.; et al. Differential microRNA Expression in USP8-Mutated and Wild-Type Corticotroph Pituitary Tumors Reflect the Difference in Protein

Ubiquitination Processes. J. Clin. Med. 2021, 10, 375. https://doi.org/ 10.3390/jcm10030375

Academic Editor: Anne Barlier Received: 13 December 2020

Accepted: 14 January 2021

Published: 20 January 2021

Publisher's Note: MDPI stays neutral with regard to jurisdictional claims in published maps and institutional affiliations.

Copyright: (C) 2021 by the authors Licensee MDPI, Basel, Switzerland. This article is an open access article distributed under the terms and conditions of the Creative Commons Attribution (CC BY) license (https:// creativecommons.org/licenses/by/ $4.0 /)$.
1 Department of Molecular and Translational Oncology, Maria Sklodowska-Curie National Research Institute of Oncology, 02-781 Warsaw, Poland; mateusz.bujko@coi.pl (M.B.); paulina.kober@gmail.com (P.K.); joanna.boresowicz@gmail.com (J.B.); natarusetska@gmail.com (N.R.); jas@coi.waw.pl (J.A.S.)

2 Department of Gastroenterology, Hepatology and Clinical Oncology, Medical Centre for Postgraduate Education, 01-813 Warsaw, Poland; natalia.zeber@o2.pl (N.Z.-L.); jostrow@warman.com.pl (J.O.)

3 Department of Neuroendocrinology, Centre of Postgraduate Medical Education, 01-813 Warsaw, Poland; agapaziewska@poczta.onet.pl

4 Department of Pathology and Laboratory Diagnostics, Maria Sklodowska-Curie Institute-Oncology Center, 02-781 Warsaw, Poland; monika.pekul@pib-nio.pl

5 Department of Neurosurgery, Military Institute of Medicine, 04-141 Warsaw, Poland; gzielinski@wim.mil.pl (G.Z.); astyk@wim.mil.pl (A.S.)

6 Department of Neurosurgery, Maria Sklodowska-Curie Institute-Oncology Center, 02-781 Warsaw, Poland; jkunickii@gmail.com

7 Department of Genetics, Maria Sklodowska-Curie Institute-Oncology Center, 02-781 Warsaw, Poland

* Correspondence: maria.maksymowicz@pib-nio.pl or mateusz.bujko@pib-nio.pl

\begin{abstract}
Background: USP8 mutations are the most common driver changes in corticotroph pituitary tumors. They have direct effect on cells' proteome through disturbance of ubiquitination process and also influence gene expression. The aim of this study was to compare microRNA profiles in USP8mutated and wild-type tumors and determine the probable role of differential microRNA expression by integrative microRNA and mRNA analysis. Methods: Patients with Cushing's disease $(n=28)$ and silent corticotroph tumors $(n=20)$ were included. USP8 mutations were identified with Sanger sequencing. MicroRNA and gene expression was determined with next-generation sequencing. Results: USP8-mutated patients with Cushing's disease showed higher rate of clinical remission and trend towards lower tumor volume than wild-type patients. Comparison of microRNA profiles of USP8-mutated and wild-type tumors revealed 68 differentially expressed microRNAs. Their target genes were determined by in silico prediction and microRNA/mRNA correlation analysis. GeneSet Enrichment analysis of putative targets showed that the most significantly overrepresented genes are involved in protein ubiquitination-related processes. Only few microRNAs influence the expression of genes differentially expressed between USP8-mutated and wild-type tumors. Conclusions: Differences in microRNA expression in corticotropinomas stratified according to USP8 status reflect disturbed ubiquitination processes, but do not correspond to differences in gene expression between these tumors.
\end{abstract}

Keywords: Cushing's disease; corticotroph PitNET; miRNA expression; gene expression; next generation sequencing; USP8; USP48; mutation

\section{Introduction}

Pituitary neuroendocrine tumors (PitNETs) represent about $10-20 \%$ of all intracranial neoplasms in adults. They may arise from various kinds of secretory cells of pituitary gland, including corticotroph cells, which produce adrenocorticotropic hormone (ACTH). Corticotroph PitNETs commonly cause ACTH-dependent Cushing's disease (CD); however, 
a notable proportion of tumors originated from corticotropic pituitary cells are endocrinologically non-functioning and classified as silent corticotroph tumors commonly referred to as silent corticotroph adenomas (SCA). Both active and silent corticotroph PitNETs share a similar molecular profile [1,2].

Recently, notable progress in the understanding of pathogenesis of $C D$ has been made [3], including the discovery of recurrent USP8 mutations [4-6]. These mutations are observed in approximately $30-40 \%$ of patients suffering from Cushing's disease as well as in silent tumors [4,6-9]. Patients with Cushing's disease with and without USP8 mutations have a slightly different clinical profile according to previously published data $[4,6,8,10-15]$. The studies showed that USP 8 mutation is related to lower tumor size $[4,5,8]$ and clinical remission after surgery [8] [12]. Additionally, differences in expression of possible molecular predictive markers as $M G M T$ or somatostatin receptor were also observed in this group of patients [2,8]. Perhaps testing the USP8 mutation status in patients could provide some kind of clinically useful information; however, the clinical results published up to today are insufficient, and molecular consequences of this mutation are only partially recognized.

USP8 gene encodes for deubiquitinase enzyme involved in the regulation of proteasomal protein degradation. USP8 mutations are small, single codon deletions or missense variants that occur in the region involved in binding 14-3-3 proteins family members. Thus, these changes impair interactions between USP8 and 14-3-3 proteins, which normally suppress deubiquitinase activity [16]. As a result of mutation, USP8 activity is enhanced and leads to preventing proteasomal degradation of particular proteins and dysregulation of natural protein turnover. This was clearly shown in in vitro experiments, providing the explanation of the sustained EGFR signaling in USP8-mutated corticotroph PitNETs [4,5]. Since USP8 deubiquitinase has many molecular substrates, its impaired functioning has potentially a wide effect on the protein level. The pleiotropic effect of the mutation is reflected by differences in gene expression between USP8-mutated (USP8mut) and USP8-wild type (USP8wt) tumors [1,2]. Accordingly, differences in expression of particular proteins related to corticotroph tumors growth were also found [15]. The understanding of the biological difference between wild type and mutated tumors appears important, since USP8 mutation may potentially serve as prognostic and predicting factor [2].

The aim of this study was to compare the profiles of microRNA (miRNA) expression in corticotroph tumors stratified according to USP8 mutational status and to determine the potential role of differential miRNA expression. Moreover, mutations of another deubiquitinase-encoding gene (USP48) contribute to pathogenesis of some USP8wt tumors $[17,18]$. Mutations of both genes were determined and taken into account in data analysis.

\section{Experimental Section}

\subsection{Patients and Samples}

Pituitary tumor samples were collected during transsphenoidal surgery and fixed in formalin for routine diagnostic procedures, including immunohistochemical and ultrastructural evaluation. Archival formalin-fixed paraffin-embedded (FFPE) tissue samples from 48 patients, including 28 samples from patients with Cushing's disease and 20 samples from patients with SCA from years 2013-2017, were included. Patients were diagnosed according to WHO criteria applied during the time of tissue sampling [19]. Diagnosis was based on results of immunohistochemical staining for pituitary hormones and Ki-67 labeling as well as commonly accepted ultrastructural features of corticotroph tumors [20]. For this study, all the tumor samples were reevaluated histopathologically by one pathologist to confirm the diagnosis and determine tumor tissue content within each sample.

The diagnosis of Cushing's syndrome/hypercortisolism was based on standard hormonal criteria: increased urinary free cortisol (UFC) in three $24 \mathrm{~h}$ urine collections, disturbances of cortisol circadian rhythm, increased serum cortisol levels accompanied by increased or not suppressed plasma ACTH levels at 8 a.m., and a lack of suppression of serum cortisol levels to $<1.8 \mu \mathrm{g} / \mathrm{dL}$ during an overnight dexamethasone suppression 
test ( $1 \mathrm{mg}$ at midnight). The pituitary etiology of Cushing's disease was confirmed based on the serum cortisol levels or UFC suppression $<50 \%$ with a high-dose dexamethasone suppression test ( $2 \mathrm{mg}$ q.i.d. (lat. quater in die $=$ four time a day) for $48 \mathrm{~h}$ ) or a positive result of a corticotrophin-releasing hormone stimulation test (100 mg i.v. (intravenously)) and positive pituitary magnetic resonance imaging. In the group of SCAs, none of the patients had any evidence of hypercortisolism based on clinical signs and symptoms as well as basic laboratory tests. ACTH levels were assessed using IRMA (immunoradiometric assay) (ELSA-ACTH, CIS Bio International, Gif-sur-Yvette Cedex, France). The analytical sensitivity was $2 \mathrm{pg} / \mathrm{mL}$ (reference range: 10-60 pg/mL). Serum cortisol concentrations were determined by the Elecsys 2010 electrochemiluminescence immunoassay (Roche Diagnostics, Mannheim, Germany). Analytical sensitivity of the assay was $0.02 \mu \mathrm{g} / \mathrm{dL}$ (reference range: 6.2-19.4 $\mu \mathrm{g} / \mathrm{dL}$ ). UFC was determined after extraction (liquid/liquid with dichloromethane) by electrochemiluminescence immunoassay (Elecsys 2010, Roche Diagnostics, Mannheim, Germany)_reference range: $4.3-176 \mu \mathrm{g} / 24 \mathrm{~h}$. Bilateral inferior petrosal sinus sampling was used as a routine investigation tool in any patient with proven ACTH-dependent Cushing's syndrome and negative or equivocal MRI findings (intrasellar lesion $\leq 6 \mathrm{~mm}$ ) [21]). A macroadenoma was defined as a tumor with at least one diameter exceeding $10 \mathrm{~mm}$, and the tumor volume was assessed with the diChiro Nelson formula (height $\times$ length $\times$ width $\times \pi / 6$ ). Invasive growth of the tumors was evaluated using Knosp grading [22]. Tumors with Knosp grades 0, 1 and 2 were considered non-invasive, while those with Knosp 3 and 4 were considered invasive.

Overall characteristics of the patients are presented in Table 1 while details are provided in Table S1. The content of tumor tissue in each FFPE sample ranged between 80 and 100\% (median 99\%) (details in Table S1). The study was approved by the local Ethics Committee of Maria Sklodowska-Curie Institute-Oncology Center in Warsaw, Poland. Each patient provided informed consent for the use of tissue samples for scientific purposes.

DNA and total RNA from FFPE samples was isolated using RecoverAll ${ }^{\mathrm{TM}}$ Total Nucleic Acid Isolation Kit for FFPE (Thermo Fisher Scientific, Waltham, MA, USA) and measured using NanoDrop 2000 (Thermo Fisher Scientific, Waltham, MA, USA). The samples were stored at $-70{ }^{\circ} \mathrm{C}$.

\subsection{Genomic Mutation Testing}

The presence of point mutation at the USP8 hotspot (exon 14) and USP48 hotspot (exon 10) was determined using Sanger sequencing. DNA was PCR amplified with FastStart Taq DNA Polymerase (Roche Diagnostics, Mannheim, Germany) using the GeneAmp 9700 PCR system (Applied Biosystems, Foster City, CA, USA). The PCR product was purified using ExoSAP-IT (USA Affymetrix, Cleveland, OH, USA), labeled with BigDye Terminator v.3.1 (Applied Biosytems, Foster City, CA, USA) according to the manufacturer's instructions and analyzed by capillary electrophoresis with the ABI PRISM 3300 Genetic Analyzer (Applied Biosystems, Foster City, CA, USA), as described previously [1]. The following sequences of PCR primers were used: $5^{\prime}$-TCCACCCCTCCAACTCATAA and $5^{\prime}$-CTGACAGATTCAGAGTAGAAACT for USP8 mutation testing as well as $5^{\prime}$ GCCCGGCTAAAGAATAAACA and 5'-TGCCTGCTATAATCCTGGAAA for identification of USP48 variants.

\subsection{Determining miRNA Expression Profile with Next Generation Sequencing (NGS)}

The quality of small RNA fractions was assessed using Agilent 2100 Bioanalyzer with Small RNA Kit chip (Agilent, Santa Clara, CA, USA) and measured with Qubit RNA HS Assay Kits (Thermo Fisher Scientific, Waltham, MA, USA). One $\mu g$ of total RNA was used to sequencing library construction with an Ion Total RNA-Seq Kit v2 (Thermo Fisher Scientific), according to the manufacturer's protocol. Ion Xpress ${ }^{\mathrm{TM}}$ RNA-Seq Barcode Kit, which allows for multiplexed sequencing, was used for hybridization and ligation of RNA adapters. RNA reverse transcription and subsequent cDNA purification and library size selection were performed using Nucleic Acid Binding Beads. cDNA was PCR-amplified, 
followed by DNA purification and size selection. Amount and size distribution of the amplified DNA was determined using Bioanalyzer 2100 using a High Sensitivity DNA Kit (Agilent, Santa Clara, CA, USA). The length of miRNA ligation products in barcoded libraries ranged between 94 and $114 \mathrm{bp}$. Template preparation for clonal amplification of up to four miRNA libraries at a concentration of $18 \mathrm{pM}$, and loading of the PI chip, were performed using Ion Chef instrument, with Ion $\mathrm{PI}^{\mathrm{TM}} \mathrm{Hi}-\mathrm{Q}^{\mathrm{TM}}$ Chef Kit (Thermo Fisher Scientific, Waltham, MA, USA). An Ion Proton sequencer (Thermo Fisher Scientific, Waltham, MA, USA) was used for sequencing. Unmapped bam files were converted into fastq files with a bamToFastq script from bedtools. Read mapping to known human miRNAs (according to miRBase release 22) and reads quantification were performed using miRDeep2.14. Data normalization and differential expression analysis were performed using DESeq2. Filtration for low-expression miRNAs and miRNAs genes with less than five sequencing reads in at least half of the samples were excluded. Fold change of expression (FC) calculated as ratio of the normalized read-count value in USP8-mutated and USP8-wt tumors was used as a measure of expression difference. Differentially expressed miRNAs were defined as those with $|\mathrm{FC}|>2$ and adjusted $p$-value $<0.05$.

\subsection{Gene Expression Profiling}

Gene expression profiles were determined in 24 FFPE samples of corticotroph tumors by sequencing of amplicon-based library representing whole transcriptome, as described in detail previously [1]. Ion AmpliSeq ${ }^{\mathrm{TM}}$ Transcriptome Human Gene Expression Kit (Thermo Fisher Scientific, Waltham, MA, USA) was used for library preparation and semiconductive sequencing technology with Ion Proton instrument, PI chip, and the sequencing reagents included in Ion $\mathrm{PI}^{\mathrm{TM}} \mathrm{Hi}-\mathrm{Q}^{\mathrm{TM}}$ Sequencing 200 Kit according to the manufacturer's instructions (Thermo Fisher Scientific, Waltham, MA, USA). Data processing was performed using Bioconductor packages in $R$ environment as described [1]. Differentially expressed genes (DEGs) were defined as those with adjusted $p$-value $<0.05$.

\subsection{Prediction of miRNA-mRNA Interactions}

Analysis of the interactions between miRNAs and mRNAs was applied to determine the possible functional role of miRNAs, which are differentially expressed in USP8mut and USP8wt tumors. We used both mRNA target prediction and correlation analysis of the expression levels of particular miRNAs and their predicted mRNA targets in corticotroph tumor samples.

The MicroRNA Data Integration Portal (mirDIP) algorithm, which combines multiple sources for miRNA target prediction [23], was used for the identification of possible mRNA targets. Only mRNAs that were predicted as targets with a probability scored as VeryHigh, according to the mirDIP criterion, were taken into account and included into downstream analyses.

Then, the correlation between the expression levels of identified potentially interacting miRNAs and mRNAs was assessed using normalized read-count data from small RNA sequencing and matched data from gene expression profiling for the same tumor samples (GSE132982 dataset). Spearman rank correlation was calculated using data from expression profiling of 24 tumors in R environment. Unadjusted $p<0.01$ was considered relevant for correlation results.

\subsection{Statistical Analysis}

Datasets of quantitative variables were tested for the normal distribution with ShapiroWilk test. Variables with normal distribution were analyzed with two-sided unpaired t-test, while a two-sided Mann-Whitney U-test was used when normal distribution was not verified. Exact Fisher's test was used for the analysis of proportions. Significance threshold of $\alpha=0.05$ was adopted. For the identification of differentially expressed miRNAs and genes, $p$-values were adjusted for multiple testing with the Benjamini-Hochberg method. The Spearman correlation method was used for correlation analysis. Data were analyzed 
using GraphPad Prism 6.07 (GraphPad Software). Hierarchical clustering analysis was conducted with Cluster 3.0, and the results were visualized using TreeView 1.6 software (Stanford University School of Medicine, Stanford, CA, USA).

Table 1. Patients' characteristics.

\begin{tabular}{|c|c|}
\hline Number of Patients: & 48 \\
\hline \multicolumn{2}{|l|}{ Diagnosis (percentage of patients) } \\
\hline Cushing's disease & $58 \%(28 / 48)$ \\
\hline Silent corticotroph adenoma & $42 \%(20 / 48)$ \\
\hline \multicolumn{2}{|l|}{ Age (years) } \\
\hline Range & $23-77$ \\
\hline Median & 49 \\
\hline \multicolumn{2}{|l|}{ Gender (percentage of patients) } \\
\hline Male & $25 \%(12 / 48)$ \\
\hline Female & $75 \%(36 / 48)$ \\
\hline \multicolumn{2}{|c|}{ Ultrastructural characteristics (percentage of patients) } \\
\hline Sparsely granulated & $44 \%(21 / 48)$ \\
\hline Densely granulated & $56 \%(27 / 48)$ \\
\hline \multicolumn{2}{|c|}{ KNOSP grade (percentage of patients) } \\
\hline 0 & $16 \%(7 / 48)$ \\
\hline 1 & $44 \%(21 / 48)$ \\
\hline 2 & $19 \%(9 / 48)$ \\
\hline 3 & $10 \%(5 / 48)$ \\
\hline 4 & $12.5 \%(6 / 48)$ \\
\hline \multicolumn{2}{|l|}{ Tumor size (percentage of patients) } \\
\hline Macroadenoma & $77 \%(37 / 48)$ \\
\hline Microadenoma & $23 \%(11 / 48)$ \\
\hline \multicolumn{2}{|c|}{ Mutation status (percentage of patients) } \\
\hline USP8 mutation & $31 \%(15 / 48)$ \\
\hline USP48 mutation & $4 \%(2 / 48)$ \\
\hline
\end{tabular}

\section{Results}

\subsection{USP8 and USP 48 Mutations}

The incidence of hotspot mutations in USP8 and USP48 was determined with Sanger sequencing in 48 patients. USP8 mutations were identified in 15/48 (35.4\%) patients. USP48 mutations were identified in $2 / 48(4.2 \%)$ patients; both were female patients suffering from Cushing's disease with a diagnosis of densely granulated corticotroph tumors. One of the tumors was microadenoma while the other was macroadenoma. Both USP48 mutations had p.Met415Ile substitution. USP8 and USP48 mutations were mutually exclusive. Details of the results are presented in Table S1. The possible relationship between the incidence of USP8 mutations and demographic/clinical parameters was investigated in groups of Cushing's disease patients and SCA patients separately. Since only two patients with USP48 mutation were identified, they were excluded from the analysis. No difference in age of diagnosis was observed between USP8-mutated (USP8mut) and USP8-wild type (USP8wt) patients, both in the Cushing's disease group and in SCA group. Except for one male patient, all USP8 mutations were identified in females; however, differences 
of proportions did not reach statistical significance in the Cushing's disease group or SCA group.

All USP8mut patients suffering from Cushing's disease were in clinical remission after surgery, while clinical remission was observed in $9 / 15$ of USP8wt patients. Difference of proportions of patients with/without remission was significant $(11 / 0$ vs. 9/6; $p=0.0237)$. A trend towards lower tumor volume was observed in USP8mut patients vs. USP $8 \mathrm{wt}$ patients in both the Cushing's disease group (median $445 \mathrm{~mm}^{3}$ vs. $2730 \mathrm{~mm}^{3}$, respectively; $p=0.0798$ ) and SCA group (median $1844 \mathrm{~mm}^{3}$ vs. $3893 \mathrm{~mm}^{3}$, respectively; $p=0.1707$ ), but no significant difference was observed. Patients suffering from Cushing's disease stratified according to USP8 mutations status did not differ in terms of preoperative clinical parameters: morning serum ACTH level, morning serum cortisol level, or $24 \mathrm{~h}$ UFC.

Among patients with silent corticotroph tumors, significantly higher $24 \mathrm{~h}$ UFC level was observed in patients with USP8 mutations than in USP8wt patients (median 124.4 vs. 66.32, respectively; $p=0.0334$ ). No difference in morning serum ACTH level, morning serum cortisol level, or midnight serum cortisol level were observed between these patients. We did not observe any difference between USP8mut and USP8wt patients in invasive growth status as determined with Knosp grading, proliferation index, or histological subtype (sparsely vs. densely granulated) either in the group of Cushing's disease patients or in those with silent tumors. The results are presented in Table 2.

Table 2. Summary of clinical features in patients with Cushing's disease and silent corticotroph tumors regarding USP8 mutation status.

\begin{tabular}{|c|c|c|c|c|c|c|}
\hline \multirow[t]{2}{*}{ Clinical Feature } & \multicolumn{3}{|c|}{ Cushing's Disease } & \multicolumn{3}{|c|}{ Silent Corticotroph Tumors } \\
\hline & USP8-mutated & USP8-wild type * & $p$-value & USP8-mutated & USP8-wild type & $p$-value \\
\hline Number of patients & $n=11$ & $n=15$ & & $n=4$ & $n=16$ & \\
\hline $\begin{array}{c}\text { Sex (ratio } \\
\text { females/males) }\end{array}$ & $10 / 1$ & $13 / 2$ & $1.00^{\mathrm{a}}$ & $4 / 0$ & $9 / 7$ & $0.2487^{\mathrm{a}}$ \\
\hline $\begin{array}{l}\text { Age at surgery } \\
\text { (years; median } \\
\text { (range)) }\end{array}$ & $36(23-67)$ & $48(24-76)$ & $0.3993^{b}$ & $41.5(23-77)$ & $54.5(34-77)$ & $0.394^{c}$ \\
\hline $\begin{array}{l}\text { Cortisol 08:00 h } \\
(\mu \mathrm{g} / \mathrm{dL} ; \text { median } \\
(\text { range }))\end{array}$ & $26.3(21-49.7)$ & $26.4(11.9-38.6)$ & $0.6036^{\mathrm{c}}$ & $16.75(9.1-50.8)$ & $18.15(6.8-29.7)$ & $0.7408^{c}$ \\
\hline $\begin{array}{l}\text { ACTH 08:00 h } \\
\text { (pg/dL; median } \\
\quad(\text { range)) }\end{array}$ & $48.2(37.3-102)$ & $82.3(36.9-129)$ & $0.1945^{c}$ & $47.7(42.1-61.3)$ & $49(14.7-74.9)$ & $0.7408^{c}$ \\
\hline $\begin{array}{l}\text { UFC ( } \mu \mathrm{g} / 24 \mathrm{~h} ; \\
\text { median (range)) }\end{array}$ & $490(276-810)$ & 497 (215-739) & $0.7974^{b}$ & $124(94.76-139)$ & $66.32(13.70-126)$ & $0.0334^{c}$ \\
\hline $\begin{array}{l}\text { Tumor volume } \\
\left(\mathrm{mm}^{3} ; \text { median }\right. \\
(\text { range }))\end{array}$ & $445.5(32-6750)$ & $2730(62.5-6000)$ & $0.0798^{c}$ & $1844(900-7350)$ & $3893(1080-11088)$ & $0.1707^{c}$ \\
\hline $\begin{array}{l}\text { Invasive tumor } \\
\text { growth (Knosp } \\
\text { grade ratio 0, I, } \\
\quad \text { II/III, IV) }\end{array}$ & $3 / 8$ & $4 / 11$ & $1.0000^{\mathrm{a}}$ & $4 / 0$ & $12 / 4$ & $0.5377^{\mathrm{a}}$ \\
\hline $\begin{array}{l}\text { Proliferation index } \\
\quad \text { (ratio Ki67 } \geq \\
3 \% / \mathrm{Ki} 67<3 \%)\end{array}$ & $4 / 7$ & $3 / 12$ & $0.4065^{\mathrm{a}}$ & $1 / 3$ & $4 / 12$ & $1.0000^{\mathrm{a}}$ \\
\hline Clinical remission & $11 / 0$ & $9 / 6$ & $0.0237^{\mathrm{a}}$ & - & - & - \\
\hline $\begin{array}{c}\text { Histology (ratio } \\
\text { sparsely/densely } \\
\text { granulated) }\end{array}$ & $3 / 8$ & $6 / 9$ & $0.6828^{a}$ & $2 / 2$ & $10 / 6$ & $1.0000^{\mathrm{a}}$ \\
\hline
\end{tabular}

* Two patients with USP48 mutations were excluded; ${ }^{a}$ indicates the use of exact Fisher's test; ${ }^{\mathrm{b}}$ indicates the use of a two-sided unpaired t-test ${ }^{c}$ indicates the use of a Mann-Whitney U-test.

\subsection{Comparing miRNA Expression in USP8mut and USP8wt Corticotroph Tumors}

The entire collection of 48 corticotroph tumors was subjected to miRNA expression profiling with next-generation sequencing of a small RNA fraction. Sequencing of small RNA libraries produced approximately 2,497,367 reads per sample, which were mapped to the human genome (hg19) and used for quantification of expression levels of known 
miRNAs, according to miRBase 22 release. Sequencing reads were annotated to 1917 miRNAs. Measurements of 1902 mature miRNAs were included in the analysis, after filtering out the miRNAs with low expression.

The overall analysis of the entire dataset with Principal Component Analysis (PCA) and hierarchical clustering methods did not show a clear separation between the groups of tumor samples stratified according to the mutation status, which indicates that the differences are not as pronounced as previously observed differences in gene expression profiles of USP8mut and USP8wt tumors [1,2]. Principal components 1 and 2 are presented in Figure S1a, while a dendrogram showing similarity of miRNA expression between the samples is shown in Figure S1b. Forty-six tumor samples were used for identification miRNAs differentially expressed in USP8mut and USP8wt tumors. Two samples with USP48 mutations were excluded from differential analysis to avoid bias resulting from possibly different molecular features of these tumors. A total of 250 differentially expressed miRNAs were found (adjusted $p$-value $<0.05$ ), including 68 miRNAs that met the criterion of $|\mathrm{FC}|>2$, as shown in Figure 1a,b. Most of them (57/68) were miRNAs with the expression higher in USP8mut than in wild-type tumors (Figure 1b).

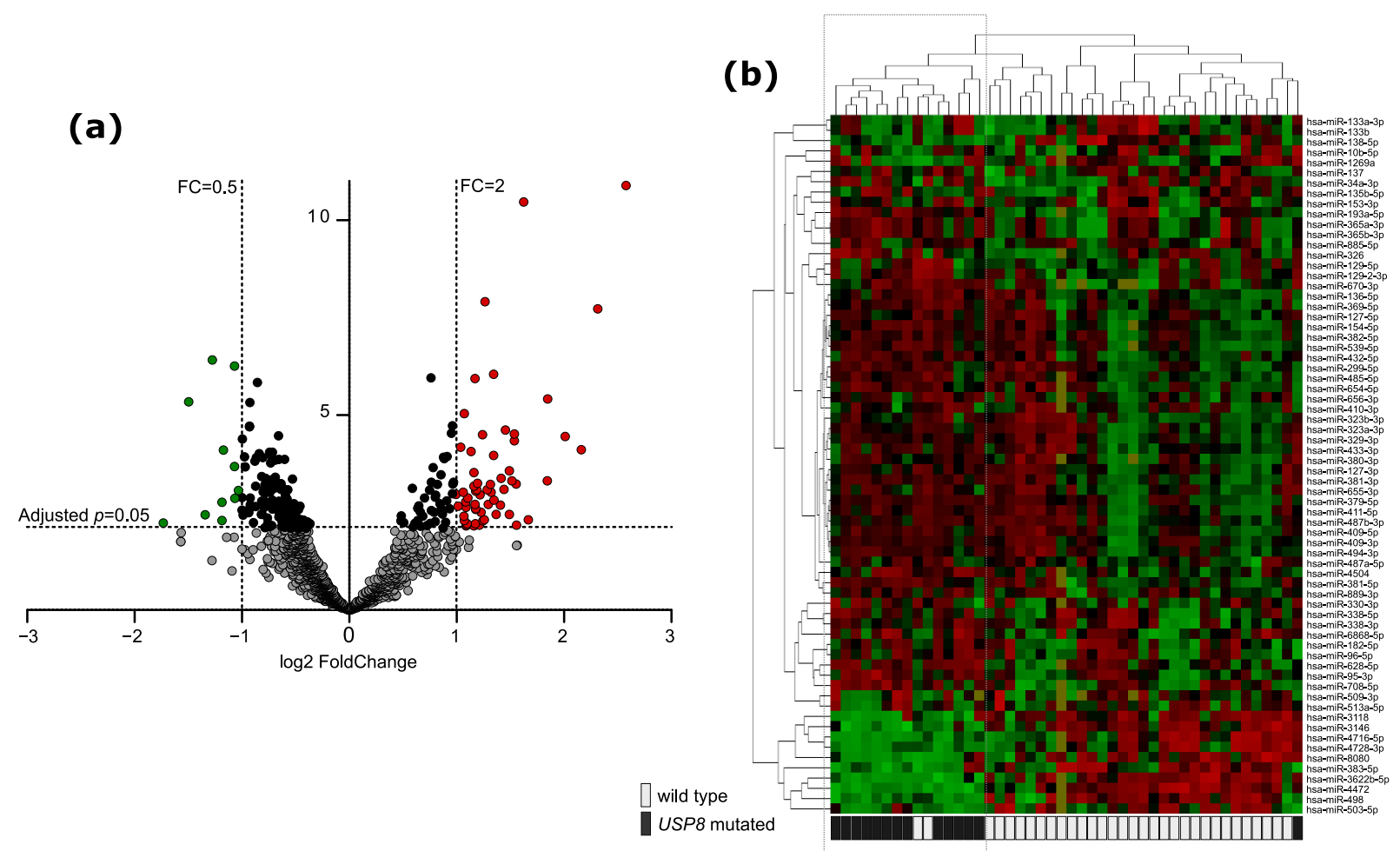

Figure 1. Difference in miRNA expression between USP8-mutated and USP8 wild-type corticotroph PitNETs. (a) Volcano plot showing differentially expressed miRNAs. Significance and fold change thresholds are marked with dashed lines. (b) The expression levels of differentially expressed miRNAs in tumor samples stratified according to USP8 mutation status with hierarchical clustering of the samples.

\subsection{Putative mRNA Targets for Differentially Expressed miRNAs}

To identify the mRNA targets of 68 differentially expressed miRNAs, a two-step procedure was applied. First, miRNA-mRNA interactions were predicted with the use of an mirDIP tool [23], and subsequently, the correlation analysis of matched miRNA and gene expression profiles was applied. This analysis included 24 tumor samples. For 49 out of 68 miRNAs differentially expressed in USP8mut vs. USP8wt tumors, significant correlation with predicted target mRNA was observed. A total of 442 miRNA-mRNAs interactions were identified with a median of four putative target mRNAs per single miRNA particle (ranging from 1 to 38 target mRNAs). Mostly negative correlation between miRNA and 
gene expression was observed as found for 303 miRNA/mRNA pairs (range of Spearman $\mathrm{R}$ coefficient -0.575 to -0.7922 ; median -0.6182$)$. Positive correlation was observed for 139 miRNA/mRNA pairs (range of Spearman R coefficient: 0.5753 to 0.8361 median: 0.6215). Results are presented in detail in Table S3.

These analyses indicated 400 putative target genes that were identified as regulated by differentially expressed miRNAs. For the evaluation of potential functional significance of these genes, a subsequent gene set enrichment analysis (GSEA) was applied with the use of three gene ontology catalogs: KEGG Pathways, Gene Ontology (GO) Molecular Function and GO Biological Processes. Four KEGG pathways were found as significantly enriched for the putative target genes, including "Ubiquitin mediated proteolysis" as the most significantly enriched (according to $p$-value). The analysis with GO Molecular Function showed three protein ubiquitination related pathways as being in the top nine significantly enriched functions: Ubiquitin-like protein ligase activity (GO:0061659), Ubiquitin protein ligase activity (GO:0061630), and ubiquitin-protein transferase activity (GO:0004842). GO Biological process database indicates two processes related to the regulation of transcriptional activity and protein ubiquitination (GO:0016567) as the three most significantly enriched process (Figure 2a). The details of GSEA results are presented in Table S3. The genes with a clear ubiquitination function, which are common for the ubiquitination-related processes and pathways, are listed in Table 3 with details of the miRNA/mRNA correlation analysis. Since a difference in interaction patterns between miRNAs with negative and positive miRNA-gene correlation were described previously [24], GSE analyses were also performed separately for putative gene targets, the expression of which is positively or negatively correlated with DEMs. This showed no significant overrepresentation for genes with positive miRNA-mRNA correlation, while we observed a clear enrichment of protein ubiquitination-related pathways and processes for genes characterized by negative miRNA-mRNA correlation. The analysis with GO Biological process indicated a proteasome-mediated ubiquitin-dependent protein catabolic process (GO:0043161), protein polyubiquitination (GO:0000209), and protein ubiquitination (GO:0016567), while GO Molecular Function showed ubiquitin-like protein ligase activity (GO:0061659), ubiquitin protein ligase activity (GO:0061630), ubiquitin-protein transferase activity (GO:0004842), RNA binding (GO:0003723), and glucocorticoid receptor binding (GO:0035259) as significantly enriched (presented in Figure $2 \mathrm{~b}$ and in Table S3).

(a)
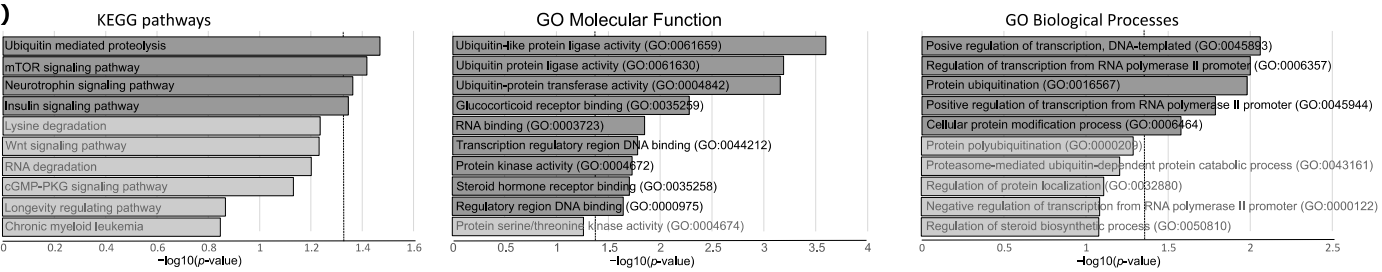

(b)

GO Molecular Function

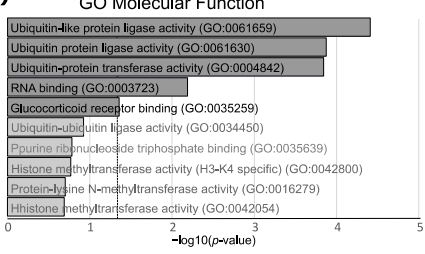

GO Biological Processes

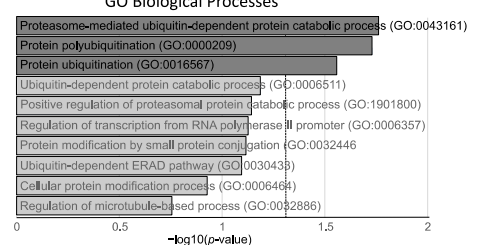

Figure 2. Gene Set Enrichment analysis of genes that were identified as regulated by miRNAs differentially expressed in USP8mut and USP8wt corticotroph tumors. (a) Results of the analysis of all putative target genes $(n=400)$. (b) Results for putative target genes with negative miRNA-mRNA correlation of expression levels $(n=239)$. Top 10 enriched pathways/processes are presented. Dark gray bars indicate significantly enriched pathways/processes (adjusted $p<0.05)$. Vertical dashed line indicates the significance threshold. 
Table 3. The list of protein ubiquitination-related genes regulated by differentially expressed miRNAs (according to target prediction and correlation analysis) that were commonly found in the significantly enriched process in GSE analysis. The results of the correlation analysis between miRNA and predicted target mRNA levels and the results of miRNA differential analysis of USP8mut and USP8wt tumors.

\begin{tabular}{|c|c|c|c|c|c|}
\hline $\begin{array}{c}\text { Differentially } \\
\text { Expressed miRNA }\end{array}$ & $\begin{array}{c}\text { Fold Change of } \\
\text { miRNA Expression }\end{array}$ & $\begin{array}{c}\text { Adjusted } \\
p \text {-Value }\end{array}$ & Predicted Target Gene & Spearman $\mathbf{R}$ & $p$-Value \\
\hline & \multicolumn{5}{|c|}{ Ubiquitin-conjugating enzyme } \\
\hline hsa-miR-182-5p & 2.11 & 0.03245 & $U B E 2 D 4$ & 0.733 & 0.000359 \\
\hline hsa-miR-133a-3p & 3.07 & 0.040107 & $U B E 2 Q 1$ & 0.720 & 0.000503 \\
\hline hsa-miR-153-3p & 2.04 & 0.022436 & $U B E 2 K$ & -0.594 & 0.007305 \\
\hline hsa-miR-96-5p & 2.09 & 0.023363 & UBE2K & -0.582 & 0.008914 \\
\hline hsa-miR-137 & 2.57 & 0.003923 & UBE2G2 & -0.592 & 0.007544 \\
\hline \multirow[t]{2}{*}{ hsa-miR-330-3p } & 2.62 & 0.00294 & UBE2J1 & -0.587 & 0.008197 \\
\hline & \multicolumn{5}{|c|}{ E3-ubiquitin ligase } \\
\hline hsa-miR-137 & 2.57 & 0.003923 & RNF165 & -0.721 & 0.000495 \\
\hline hsa-miR-382-5p & 2.71 & 0.022428 & KLHL42 & -0.704 & 0.000763 \\
\hline hsa-miR-433-3p & 2.93 & 0.011477 & FBXO22 & -0.634 & 0.003579 \\
\hline hsa-miR-127-5p & 2.14 & 0.022624 & PELI2 & -0.624 & 0.004318 \\
\hline hsa-miR-133b & 2.79 & 0.029053 & KLHL9 & 0.613 & 0.005292 \\
\hline hsa-miR-498 & 0.37 & 0.001367 & $A M F R$ & -0.603 & 0.006319 \\
\hline hsa-miR-329-3p & 2.79 & 0.006727 & PELI2 & -0.592 & 0.007635 \\
\hline hsa-miR-338-5p & 2.06 & 0.003694 & PJA2 & 0.590 & 0.007876 \\
\hline hsa-miR-153-3p & 2.04 & 0.022436 & RNF26 & -0.587 & 0.008254 \\
\hline hsa-miR-410-3p & 2.65 & 0.011477 & RNF144B & -0.585 & 0.008572 \\
\hline hsa-miR-498 & 0.37 & 0.001367 & MARCH4 & -0.583 & 0.008784 \\
\hline hsa-miR-432-5p & 2.85 & 0.00294 & KLHL20 & -0.580 & 0.009173 \\
\hline \multirow[t]{2}{*}{ hsa-miR-432-5p } & 2.85 & 0.00294 & CUL5 & -0.578 & 0.009531 \\
\hline & \multicolumn{5}{|c|}{ Deubiquitinase } \\
\hline hsa-miR-381-3p & 2.50 & 0.022163 & USP46 & -0.689 & 0.001117 \\
\hline hsa-miR-498 & 0.37 & 0.001367 & USP46 & -0.664 & 0.001953 \\
\hline
\end{tabular}

\subsection{Difference in miRNA Profile and Differential Gene Expression}

The difference in gene expression profiles between USP8mut and USP8wt tumors was determined using sequencing data for 24 tumor samples, which were also included in miRNA-mRNA correlation analysis. The results of differential analysis indicated 1648 DEGs that met the criterion of adjusted $p<0.05$. In order to identify DEGs with expression differences resulting from distinct miRNA profile in corticotroph tumors, stratified according to USP8 mutation status, we compared the results of three analyses: differential gene expression analysis, differential miRNA expression analysis, and identification of putative miRNA-mRNA interaction. DEGs with a direction of expression fold change that is concordant with the sign of correlation and the expression change of the corresponding miRNA were considered relevant. In case of genes with a negative miRNA-mRNA correlation, we looked for genes with opposite fold change signs, while in the case of genes with positive correlation, we looked for genes with the same fold change signs.

Out of the 400 target mRNAs of which the expression correlated with differentially expressed miRNAs, 25 genes had significantly different expression level in USP8mut and USP8wt tumors. This included 21 genes with negative correlation of miRNA-mRNA expression: KDM5A, KMT2C, SLAIN2, PGGT1B, RBM33, SNX13, KIAA0355, ANKRD52, PCDHAC2, CCDC88C, AFF1, GAS1, APLF, DNAJC6, RASAL2, LRP12, FAM135A, GMFB, SORT1, FAM133B and NFIA. In turn, it includes four genes with positive expression correlation: RAB15, PALM2, ELMO2, and JPH3, which were differentially expressed. These 25 DEGs are putative targets of 12 miRNAs: hsa-miR-96-5p, hsa-miR-708-5p, hsa-miR655-3p, hsa-miR-539-5p, hsa-miR-498, hsa-miR-382-5p, hsa-miR-383-5p, hsa-miR-330-3p, hsa-miR-329-3p, hsa-miR-326, hsa-miR-513a-5p, and hsa-miR-153-3p. The results are visualized in Figure 3 and presented in detail in Table 4. 


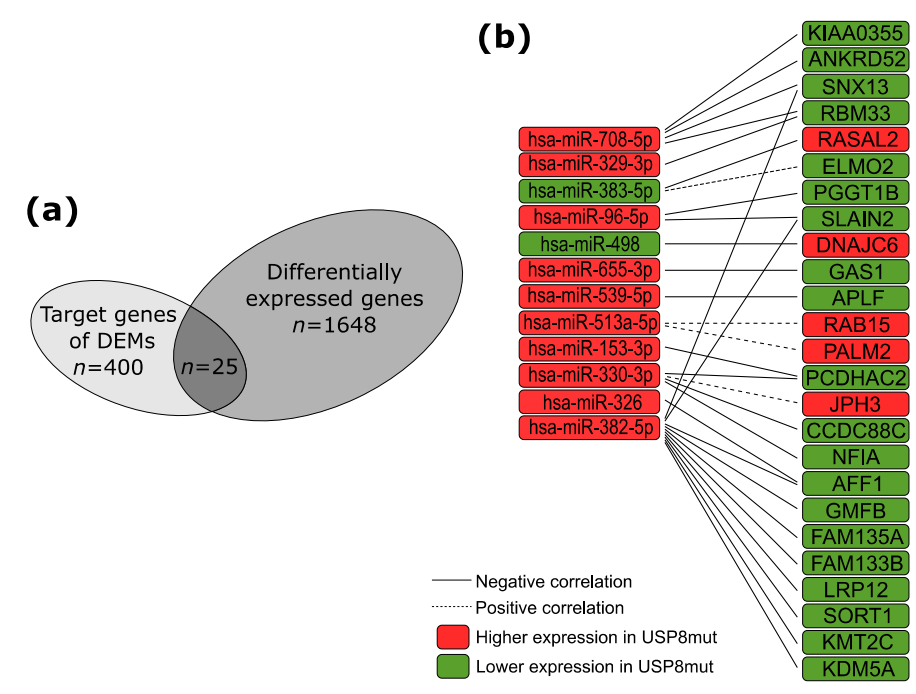

Figure 3. The predicted contribution of differentially expressed miRNAs (DEMs) in differential gene expression. (a) The proportion of genes differentially expressed in USP8mut and USP8wt corticotroph tumors; (b) genes that are putative targets of DEMs.

Table 4. Genes with distinct expression levels in USP8mut and USP8wt tumors with an expression difference related to the levels of differentially expressed miRNAs.

\begin{tabular}{|c|c|c|c|c|c|c|c|}
\hline \multirow[b]{2}{*}{ Gene } & \multirow[b]{2}{*}{ MicroRNA } & \multicolumn{2}{|c|}{ Correlation Analysis } & \multicolumn{2}{|c|}{ Differential Gene Expression } & \multicolumn{2}{|c|}{ Differential miRNA Expression } \\
\hline & & Spearman R & $p$-value & Fold change & Adjusted $p$-value & Fold change & Adjusted $p$-value \\
\hline PGGT1B & hsa-miR-96-5p & -0.739 & 0.000302 & 0.55 & 0.015538 & 2.09 & 0.023363 \\
\hline SLAIN2 & hsa-miR-96-5p & -0.689 & 0.001096 & 0.65 & 0.043609 & 2.09 & 0.023363 \\
\hline RBM33 & hsa-miR-708-5p & -0.734 & 0.000348 & 0.70 & 0.024614 & 4.84 & 0.000013 \\
\hline SNX13 & hsa-miR-708-5p & -0.700 & 0.000684 & 0.62 & 0.030738 & 4.84 & 0.000013 \\
\hline KIAA0355 & hsa-miR-708-5p & -0.664 & 0.001928 & 0.59 & 0.033981 & 4.84 & 0.000013 \\
\hline ANKRD52 & hsa-miR-708-5p & -0.617 & 0.004874 & 0.65 & 0.008329 & 4.84 & 0.000013 \\
\hline GAS1 & hsa-miR-655-3p & -0.612 & 0.005347 & 0.27 & 0.020942 & 2.17 & 0.037144 \\
\hline$A P L F$ & hsa-miR-539-5p & -0.615 & 0.005108 & 0.15 & 0.003026 & 2.77 & 0.013880 \\
\hline$R A B 15$ & hsa-miR-513a-5p & 0.601 & 0.006558 & 2.67 & 0.015333 & 2.11 & 0.006640 \\
\hline PALM2 & hsa-miR-513a-5p & 0.603 & 0.006263 & 2.34 & 0.032974 & 2.11 & 0.006640 \\
\hline DNAJC6 & hsa-miR-498 & -0.659 & 0.002133 & 1.65 & 0.0347725 & 0.37 & 0.001367 \\
\hline RASAL2 & hsa-miR-383-5p & -0.611 & 0.005419 & 1.72 & 0.035180 & 0.46 & 0.048076 \\
\hline ELMO2 & hsa-miR-383-5p & 0.680 & 0.001352 & 0.54 & 0.002362 & 0.46 & 0.048076 \\
\hline KDM5A & hsa-miR-382-5p & -0.762 & 0.000149 & 0.69 & 0.013025 & 2.71 & 0.022428 \\
\hline SNX13 & hsa-miR-382-5p & -0.729 & 0.000400 & 0.62 & 0.030738 & 2.71 & 0.022428 \\
\hline KMT2C & hsa-miR-382-5p & -0.701 & 0.000819 & 0.63 & 0.002765 & 2.71 & 0.022428 \\
\hline LRP12 & hsa-miR-382-5p & -0.698 & 0.000880 & 0.61 & 0.013395 & 2.71 & 0.022428 \\
\hline FAM135A & hsa-miR-382-5p & -0.696 & 0.000923 & 0.70 & 0.042200 & 2.71 & 0.022428 \\
\hline GMFB & hsa-miR-382-5p & -0.695 & 0.000952 & 0.65 & 0.012156 & 2.71 & 0.022428 \\
\hline SORT1 & hsa-miR-382-5p & -0.610 & 0.005543 & 0.64 & 0.017393 & 2.71 & 0.022428 \\
\hline FAM133B & hsa-miR-382-5p & -0.595 & 0.007159 & 0.42 & 0.014833 & 2.71 & 0.022428 \\
\hline$A F F 1$ & hsa-miR-382-5p & -0.584 & 0.008682 & 0.65 & 0.020921 & 2.71 & 0.022428 \\
\hline SLAIN2 & hsa-miR-382-5p & -0.583 & 0.008789 & 0.65 & 0.043609 & 2.71 & 0.022428 \\
\hline NFIA & hsa-miR-330-3p & -0.615 & 0.005067 & 0.37 & 0.010137 & 2.62 & 0.002940 \\
\hline РCDHAC2 & hsa-miR-330-3p & -0.587 & 0.008183 & 0.56 & 0.00004 & 2.62 & 0.002940 \\
\hline JPH3 & hsa-miR-330-3p & 0.580 & 0.008024 & 4.88 & 0.000058 & 2.62 & 0.002940 \\
\hline CCDC $88 \mathrm{C}$ & hsa-miR-329-3p & -0.689 & 0.001105 & 0.28 & 0.023204 & 2.79 & 0.006727 \\
\hline RBM33 & hsa-miR-329-3p & -0.594 & 0.007343 & 0.70 & 0.024614 & 2.79 & 0.006727 \\
\hline AFF1 & hsa-miR-326 & -0.662 & 0.001997 & 0.65 & 0.020921 & 2.31 & 0.000016 \\
\hline PCDHAC2 & hsa-miR-153-3p & -0.583 & 0.008848 & 0.56 & 0.036190 & 2.04 & 0.022436 \\
\hline
\end{tabular}




\section{Discussion}

Hotspot mutations in the USP8 gene, encoding ubiquitin carboxyl-terminal hydrolase 8 , are the most common driver changes in corticotroph PitNETs. They have been detected in $30-40 \%$ of patients in previous studies [4,6-8], as also observed in our cohort.

The clinical relevance of USP8 mutations was examined in few previous studies $[4,6,8,10-15]$. Mutations were identified predominantly in women $[4,5,12,13,25]$ and more frequently in younger patients according to some reports [6,14]. We found mutations nearly exceptionally in women (with only one male patient); however, due to a high overrepresentation of female patients in the study group, we cannot conclude that there is a sex-related difference. We also did not observe a relationship between mutation and age of onset.

In patients with Cushing's disease, these mutations appear related to lower tumor size and clinical remission after surgery $[8,12]$, which could allow to consider USP8 mutations a possible favorable prognosis marker. However, they are also related to a higher risk of recurrence [14]. In concordance with these previous observations, we found a significantly higher rate of clinical remission in USP8mut patients suffering from Cushing's disease and a trend of lower tumor volume in mutated patients; however, no statistically significant difference was determined. We did not find differences in preoperative 24-h urinary free cortisol, which was observed to be significantly higher in patients with mutations in some previous studies $[6,14]$. Moreover, we did not find the difference in the proportion of tumors with invasive growth reported in other published results [5].

The analysis of the role of USP8 mutations in silent corticotroph tumors has not been reported previously. The mutations are less frequent in this group of patients than in the case of Cushing's disease. We identified the mutations in $20 \%$ of patients, while the mutation rate was $10 \%$ in the other study by Castellnoum, which included 20 SCAs [9]. No SCA with mutation was found in a study that included 11 such patients [6]. Because only four mutated SCAs were included in the analysis, the results may only be treated as preliminary. The only clinical parameter that we found significantly different in SCA patients with and without mutation was $24 \mathrm{~h} \mathrm{UFC,} \mathrm{which} \mathrm{was} \mathrm{significantly} \mathrm{higher} \mathrm{in}$ USP8mut patients; however, it was still within the reference range.

The generalization of our results should be done cautiously, and we have to emphasize an important limitation of our clinical data analysis. The numbers of patients included in the analysis are probably too low to draw a firm conclusion and the group is not representative of the general population, especially in the case of patients suffering from Cushing's disease. Since the primary aim of our study was molecular profiling of tumor tissue, we intentionally preselected large tumors, which allowed us to have enough tissue for DNA/RNA isolation and successful molecular procedures.

From a molecular biology point of view, USP8 mutations cause deregulation of the protein polyubiquitination/deubiquitination balance and impair the normal proteasomal degradation process [16]. In corticotroph tumors, a sustained EGFR signaling was found to be a consequence of the mutation $[4,5]$ and the USP 8 changes probably affect normal turnover of many other proteins regulated by ubiquitin carboxyl-terminal hydrolase 8 [26]. The pleiotropic effect of the mutation is observed not only at the level of protein degradation, but it is also manifested at the level of gene expression [1,2]. In this study, we aimed to characterize the difference in miRNA profile in tumors with USP8 mutations and wild-type PitNETs.

Recently, a novel driver mutation in another deubiquitinating enzyme i.e., USP48, was found in patients negative for USP8 changes. USP48 pathogenic variants cause increased activity of encoded deubiquitinase against its substrates Gli1 and H2A [17]. Because USP48 mutations affect the processes of protein degradation similarly to mutations of USP8, we screened the tumor samples for both the mutations. Two samples with USP48 Met/Val variant were identified and excluded from differential miRNA analysis to avoid a bias resulting from similarities in pathogenic mechanism. Due to a very low number of USP48-mutated tumors, these patients were also excluded from the analysis of clinical data. 
We found that USP8mut and USP8wt tumors differ in miRNA expression; however, the differences are less pronounced than previously reported differences in mRNA expression [1,2]. We did not observe a clear distinction of USP8mut and USP8wt tumors in overall analysis, including PCA or hierarchical clustering based on the entire set of miRNA sequencing data, while a pronounced difference in mRNA expression profiles between USP8mut and USP8wt was reported previously [1,2].

USP8mut and USP8wt tumors differ in levels of relatively small proportion of all miRNAs that were included in differential analysis (approximately 3.5\%). Conversely, a much higher proportion of differentially expressed protein-coding $\mathrm{mRNAs}$ was identified in corticotroph tumors stratified according USP8 mutational status [1,2].

Since miRNAs play a regulatory role in expression and translation [27], the consequence of differential miRNAs' expression depends on particular mRNA targets. To determine the possible functional role of DEMs in a high-throughput approach covering multiple miRNA-mRNA interactions, we predicted the putative mRNA targets for each DEM, followed by calculating the correlation coefficient for the expression levels of matched miRNAs and mRNAs. With this procedure, we mostly identified miRNA-mRNA pairs with negative correlation between expression levels where a high level of a particular miRNA corresponded with a low expression of its target gene. This relationship is concordant with a generally accepted concept that miRNAs are negative regulators of gene expression. Still, a notable part of the identified putative target mRNAs showed positive miRNA-mRNA correlation, indicating an activating role of miRNA. Activating action was previously reported for many miRNAs [28]. Recently published pan-cancer analyses [24,29] reported that many of miRNAs dysregulated in human cancer are positively correlated with their target genes.

GSE analysis was applied to identify the pathways where the identified target genes are overrepresented. The results showed that target genes of DEMs, especially those with negative miRNA-mRNA correlation, are related mainly to pathways and processes of protein ubiquitination. Since direct effects of USP8 mutations are the changes at protein ubiquitination level [4-6], we believe that the different expression of miRNAs that are involved in editing ubiquitin marks may reflect this major biological difference between USP8mut and USP8wt corticotroph PitNETs. Protein ubiquitination processes are directly orchestrated by a high number of proteins belonging mainly to three classes: ubiquitinactivating enzymes (E1), ubiquitin conjugating enzymes (E2), and ubiquitin ligases (E3) [30]. The enzymes of each class catalyze the subsequent stages of protein ubiquitination and the reverse reaction of protein deubiquitination is conducted by deubiquitinating enzymes [30]. Each class of the enzymes involved in editing ubiquitin marks includes multiple proteins, and genes encoding for proteins belonging to each class were found as putative targets of DEMs.

Distinct expression of miRNAs appears to have a limited effect on differential gene expression in USP8mut and USP8wt tumors. Less than $10 \%$ of predicted target genes with correlated miRNA-mRNA expression levels have significantly different expression in corticotroph tumors with and without mutation. The mRNA level of only 25 out of over 1600 DEGs could be considered as related to a different miRNA expression. This means that factors other than miRNA are responsible for the previously described highly different gene expression profile in USP8mut and USP8wt PitNETs.

None of the ubiquitination processes-related genes that were identified as putative targets of DEMs have significantly distinct expression levels. However, some of DEGs that were identified as targets of DEMs may have an interesting role in the biology of USP8mut corticotroph PitNETs. For example, our results indicate that hsa-mir-382-5p, which has higher expression in USP8mut tumors, may regulate genes involved in transcriptional regulation: KMT2C, KDM5A, and AFF1. KMT2C encodes for lysine methyltransferase that introduces mono-methylation mark at histone H3K4 [31], while KDM5A is lysine demethylase that converts di- and trimethylated H3K4 into mono-methylated form [32]. H3K4 mono-methylation plays a role in regulation of gene enhancers activation [33]. In turn, 
AFF1 functions as a regulator of transcription elongation and chromatin remodeling [34]. This suggests that hsa-mir-382-5p may contribute to a large difference in gene expression levels between USP8mut and USP8wt corticotrophinomas.

Our results also suggest that hsa-miR-655-3p, which has higher expression in mutated tumors, may affect expression levels of GAS1. The protein encoded by this gene is a negative regulator of the cell cycle and is considered a tumor suppressor in gastric and colorectal cancer [35,36]. It is known that corticotroph PitNETs with a distinct USP8 status differ in the expression status of cell cycle regulators at gene and protein level [1,2,15].

It is worth emphasizing that miRNA-mRNA interaction analysis results based on target prediction and calculation of expression correlation should be treated as preliminary. The commonly used methodology of detail validation of miRNA-mRNA interactions utilize laborious experimenting in vitro to confirm the impact of miRNA level on target gene expression and confirmation of physical miRNA-mRNA interaction with luciferase assay. This wet-lab approach is practically unfeasible for simultaneous investigation of many target genes of multiple miRNAs that we attempted to perform in our study. Additionally, this approach requires an appropriate cell model, but no human cell line of corticotroph cells is available. The only stable line of corticotroph cells are mouse AtT-20 cells and its usefulness in investigation of miRNA-mRNA interaction in human is limited due to evolutionary differences between species [37]. Some data on miRNA function in corticotroph cells based on mouse cell line were published [38-40]; however, it must be taken into account that approximately $46 \%$ miRNAs are considered primate-specific, while $14 \%$ are human-specific [37].

In summary, in our study we compared miRNA profiles of USP8mut and USP8wt corticotroph PitNETs and determined miRNAs with different expression levels. With target prediction and comprehensive miRNA and mRNA expression data analysis, we found that putative targets of DEMs are mainly the genes involved in processes and pathways of protein ubiquitination. However, differences in only a few miRNAs appear to affect the levels of genes with significantly diverse expression in corticotrophinomas with and without USP8 mutations. Thus, the difference in miRNA levels is not the cause of a pronounced differences in the gene expression between these tumors.

Supplementary Materials: The following are available online at https:/ / www.mdpi.com/2077-038 3/10/3/375/s1. Table S1: Detail patients' characteristics, Table S2: MiRNAs differentially expressed in USP8mut and USP8wt tumors, Table S3: Identification of putative mRNA targets of miRNAs differentially expressed in USP8 mutated and wild type tumors by target prediction and correlation analysis, Table S4: Top 10 pathways enriched for the genes that are targets of differentially expressed miRNAs (based on in silico target prediction and miRNA/mRNA correlation analysis); Figure S1: The overall analysis of corticotroph tumor samples stratified according to USP8 mutation status based on the entire miRNA dataset. a) Principal Component Analysis; b) Hierarchical clustering.

Author Contributions: Conceptualization, M.M. and M.B.; Methodology, M.B. and J.B.; Software, J.B.; Formal analysis, P.K., J.B., and M.B.; investigation, P.K., N.R., A.P., N.Z.-L., M.P., and M.B.; resources, J.K., G.Z., A.S., and M.M.; data curation, J.B. and M.B.; writing-original draft preparation, M.B., P.K., and M.M.; writing-review and editing, all the authors; visualization, M.B. and J.B.; supervision, M.M. and J.A.S.; project administration M.M.; funding acquisition, J.O. All authors have read and agreed to the published version of the manuscript.

Funding: Supported by the Maria Sklodowska-Curie Institute-Oncology Center internal grant SN/GW09/2017.

Institutional Review Board Statement: The study was conducted according to the guidelines of the Declaration of Helsinki and approved by the local Ethics Committee of Maria SklodowskaCurie Institute-Oncology Center in Warsaw, Poland (approval number 44/2018, date of approval 26.07.2018).

Informed Consent Statement: Informed consent was obtained from all subjects involved in the study. 
Data Availability Statement: Results of small RNA sequencing were submitted to Gene Expression Omnibus database.

Conflicts of Interest: The authors declare no conflict of interest.

\section{References}

1. Bujko, M.; Kober, P.; Boresowicz, J.; Rusetska, N.; Paziewska, A.; Dabrowska, M.; Piaścik, A.; Pȩkul, M.; Zieliński, G.; Kunicki, J.; et al. USP8 mutations in corticotroph adenomas determine a distinct gene expression profile irrespective of functional tumour status. Eur. J. Endocrinol. 2019, 181, 615-627. [CrossRef] [PubMed]

2. Neou, M.; Villa, C.; Armignacco, R.; Jouinot, A.; Raffin-Sanson, M.L.; Septier, A.; Letourneur, F.; Diry, S.; Diedisheim, M.; Izac, B.; et al. Pangenomic Classification of Pituitary Neuroendocrine Tumors. Cancer Cell 2020, 37, 123-134.e5. [CrossRef] [PubMed]

3. Albani, A.; Theodoropoulou, M.; Reincke, M. Genetics of Cushing's disease. Clin. Endocrinol. (Oxf). 2018, 88, 3-12. [CrossRef] [PubMed]

4. $\quad$ Reincke, M.; Sbiera, S.; Hayakawa, A.; Theodoropoulou, M.; Osswald, A.; Beuschlein, F.; Meitinger, T.; Mizuno-Yamasaki, E.; Kawaguchi, K.; Saeki, Y.; et al. Mutations in the deubiquitinase gene USP8 cause Cushing's disease. Nat. Genet. 2015, 47, 31-38. [CrossRef] [PubMed]

5. Ma, Z.-Y.; Song, Z.-J.; Chen, J.-H.; Wang, Y.-F.; Li, S.-Q.; Zhou, L.-F.; Mao, Y.; Li, Y.-M.; Hu, R.-G.; Zhang, Z.-Y.; et al. Recurrent gain-of-function USP8 mutations in Cushing's disease. Cell Res. 2015, 25, 306-317. [CrossRef]

6. $\quad$ Perez-Rivas, L.G.; Theodoropoulou, M.; Ferraù, F.; Nusser, C.; Kawaguchi, K.; Stratakis, C.A.; Rueda Faucz, F.; Wildemberg, L.E.; Assié, G.; Beschorner, R.; et al. The gene of the ubiquitin-specific protease 8 is frequently mutated in adenomas causing cushing's disease. J. Clin. Endocrinol. Metab. 2015, 100, E997-E1004. [CrossRef]

7. Faucz, F.R.; Tirosh, A.; Tatsi, C.; Berthon, A.; Hernández-Ramírez, L.C.; Settas, N.; Angelousi, A.; Correa, R.; Papadakis, G.Z.; Chittiboina, P.; et al. Somatic USP8 gene mutations are a common cause of pediatric cushing disease. J. Clin. Endocrinol. Metab. 2017, 102, 2836-2843. [CrossRef]

8. Hayashi, K.; Inoshita, N.; Kawaguchi, K.; Ardisasmita, A.I.; Suzuki, H.; Fukuhara, N.; Okada, M.; Nishioka, H.; Takeuchi, Y.; Komada, M.; et al. The USP8 mutational status may predict drug susceptibility in corticotroph adenomas of Cushing's disease. Eur. J. Endocrinol. 2016, 174, 213-226. [CrossRef]

9. Castellnou, S.; Vasiljevic, A.; Lapras, V.; Raverot, V.; Alix, E.; Borson-Chazot, F.; Jouanneau, E.; Raverot, G.; Lasolle, H. Sst5 expression and usp8 mutation in functioning and silent corticotroph pituitary tumors. Endocr. Connect. 2020, 9, 243-253. [CrossRef]

10. Martins, C.S.; Camargo, R.C.; Coeli-Lacchini, F.B.; Saggioro, F.P.; Moreira, A.C.; De Castro, M. USP8 Mutations and Cell Cycle Regulation in Corticotroph Adenomas. Horm. Metab. Res. 2020, 52, 117-123. [CrossRef]

11. Ballmann, C.; Thiel, A.; Korah, H.E.; Reis, A.-C.; Saeger, W.; Stepanow, S.; Köhrer, K.; Reifenberger, G.; Knobbe-Thomsen, C.B.; Knappe, U.J.; et al. USP8 Mutations in Pituitary Cushing Adenomas-Targeted Analysis by Next-Generation Sequencing. J. Endocr. Soc. 2018, 2, 266-278. [CrossRef] [PubMed]

12. Wanichi, I.Q.; de Paula Mariani, B.M.; Frassetto, F.P.; Siqueira, S.A.C.; de Castro Musolino, N.R.; Cunha-Neto, M.B.C.; Ochman, G.; Cescato, V.A.S.; Machado, M.C.; Trarbach, E.B.; et al. Cushing's disease due to somatic USP8 mutations: A systematic review and meta-analysis. Pituitary 2019, 22, 435-442. [CrossRef] [PubMed]

13. Losa, M.; Mortini, P.; Pagnano, A.; Detomas, M.; Cassarino, M.F.; Pecori Giraldi, F. Clinical characteristics and surgical outcome in USP8-mutated human adrenocorticotropic hormone-secreting pituitary adenomas. Endocrine 2019, 63, 240-246. [CrossRef] [PubMed]

14. Albani, A.; Pérez-Rivas, L.G.; Dimopoulou, C.; Zopp, S.; Colón-Bolea, P.; Roeber, S.; Honegger, J.; Flitsch, J.; Rachinger, W.; Buchfelder, M.; et al. The USP8 mutational status may predict long-term remission in patients with Cushing's disease. Clin. Endocrinol. (Oxf). 2018, 89, 454-458. [CrossRef]

15. Weigand, I.; Knobloch, L.; Flitsch, J.; Saeger, W.; Monoranu, C.M.; Höfner, K.; Herterich, S.; Rotermund, R.; Ronchi, C.L.; Buchfelder, M.; et al. Impact of USP8 Gene Mutations on Protein Deregulation in Cushing Disease. J. Clin. Endocrinol. Metab. 2019, 104, 2535-2546. [CrossRef]

16. Centorrino, F.; Ballone, A.; Wolter, M.; Ottmann, C. Biophysical and structural insight into the USP8/14-3-3 interaction. FEBS Lett. 2018, 592, 1211-1220. [CrossRef]

17. Sbiera, S.; Perez-Rivas, L.G.; Taranets, L.; Weigand, I.; Flitsch, J.; Graf, E.; Monoranu, C.M.; Saeger, W.; Hagel, C.; Honegger, J.; et al. Driver mutations in USP8 wild-type Cushing's disease. Neuro. Oncol. 2019, 21, 1273-1283. [CrossRef]

18. Chen, J.; Jian, X.; Deng, S.; Ma, Z.; Shou, X.; Shen, Y.; Zhang, Q.; Song, Z.; Li, Z.; Peng, H.; et al. Identification of recurrent USP48 and BRAF mutations in Cushing's disease. Nat. Commun. 2018, 9, 1-9. [CrossRef]

19. Lloyd, R.V.; Kovacs, K.; Young, W.F., Jr.; Farrell, W.E.; Asa, S.L. Pituitary Tumours. In World Health Organization Classification of Tumours: Tumours of Endocrine Organs, 3rd ed.; DeLellis, R.A., Lloyd, R.V., Heitz, P.U., Eng, C., Eds.; IARC Press: Lyon, France, 2004.

20. Asa, S.L. Tumors of the Pituitary Gland; American Registry of Pathology in Collaboration with the Armed Forces Institute of Pathology: Washington, DC, USA, 2011.

21. Zampetti, B.; Grossrubatscher, E.; Ciaramella, P.D.; Boccardi, E.; Loli, P. Bilateral inferior petrosal sinus sampling. Endocr. Connect. 2016, 5, R12-R25. [CrossRef] 
22. Knosp, E.; Steiner, E.; Kitz, K.; Matula, C.; Parent, A.D.; Laws, E.R.; Ciric, I. Pituitary adenomas with invasion of the cavernous sinus space: A magnetic resonance imaging classification compared with surgical findings. Neurosurgery 1993, 33, 610-618. [CrossRef]

23. Tokar, T.; Pastrello, C.; Rossos, A.E.M.; Abovsky, M.; Hauschild, A.C.; Tsay, M.; Lu, R.; Jurisica, I. MirDIP 4.1-Integrative database of human microRNA target predictions. Nucleic Acids Res. 2018, 46, D360-D370. [CrossRef] [PubMed]

24. Tan, H.; Huang, S.; Zhang, Z.; Qian, X.; Sun, P.; Zhou, X. Pan-cancer analysis on microRNA-associated gene activation. EBioMedicine 2019, 43, 82-97. [CrossRef] [PubMed]

25. Sesta, A.; Cassarino, M.F.; Terreni, M.; Ambrogio, A.G.; Libera, L.; Bardelli, D.; Lasio, G.; Losa, M.; Pecori Giraldi, F. UbiquitinSpecific Protease 8 Mutant Corticotrope Adenomas Present Unique Secretory and Molecular Features and Shed Light on the Role of Ubiquitylation on ACTH Processing. Neuroendocrinology 2020, 110, 119-129. [CrossRef] [PubMed]

26. Dufner, A.; Knobeloch, K.P. Ubiquitin-specific protease 8 (USP8/UBPy): A prototypic multidomain deubiquitinating enzyme with pleiotropic functions. Biochem. Soc. Trans. 2019, 47, 1867-1879. [CrossRef] [PubMed]

27. Hayes, J.; Peruzzi, P.P.; Lawler, S. MicroRNAs in cancer: Biomarkers, functions and therapy. Trends Mol. Med. 2014, 20, 460-469. [CrossRef]

28. Stavast, C.J.; Erkeland, S.J. The Non-Canonical Aspects of MicroRNAs: Many Roads to Gene Regulation. Cells 2019, 8, 1465. [CrossRef]

29. Xu, P.; Wu, Q.; Yu, J.; Rao, Y.; Kou, Z.; Fang, G.; Shi, X.; Liu, W.; Han, H. A Systematic Way to Infer the Regulation Relations of miRNAs on Target Genes and Critical miRNAs in Cancers. Front. Genet. 2020, 11, 1-13. [CrossRef]

30. Gallo, L.H.; Ko, J.; Donoghue, D.J. The importance of regulatory ubiquitination in cancer and metastasis. Cell Cycle 2017, 16, 634-648. [CrossRef]

31. Sze, C.C.; Shilatifard, A. MLL3/MLL4/COMPASS family on epigenetic regulation of enhancer function and cancer. Cold Spring Harb. Perspect. Med. 2016, 6, 1-16. [CrossRef]

32. Kirtana, R.; Manna, S.; Patra, S.K. Molecular mechanisms of KDM5A in cellular functions: Facets during development and disease. Exp. Cell Res. 2020, 396, 112314. [CrossRef]

33. Local, A.; Huang, H.; Albuquerque, C.P.; Singh, N.; Lee, A.Y.; Wang, W.; Wang, C.; Hsia, J.E.; Shiau, A.K.; Ge, K.; et al Identification of H3K4me1-associated proteins at mammalian enhancers. Nat. Genet. 2018, 50, 73-82. [CrossRef]

34. Kumari, N.; Hassan, M.A.; Lu, X.; Roeder, R.G.; Biswas, D. AFF1 acetylation by p300 temporally inhibits transcription during genotoxic stress response. Proc. Natl. Acad. Sci. USA 2019, 116, 22140-22151. [CrossRef] [PubMed]

35. Wang, H.; Zhou, X.; Zhang, Y.; Zhu, H.; Zhao, L.; Fan, L.; Wang, Y.; Gang, Y.; Wu, K.; Liu, Z.; et al. Growth arrest-specific gene 1 is downregulated and inhibits tumor growth in gastric cancer. FEBS J. 2012, 279, 3652-3664. [CrossRef] [PubMed]

36. Li, Q.; Qin, Y.; Wei, P.; Lian, P.; Li, Y.; Xu, Y.; Li, X.; Li, D.; Cai, S. Gas1 inhibits metastatic and metabolic phenotypes in colorectal Carcinoma. Mol. Cancer Res. 2016, 14, 830-840. [CrossRef] [PubMed]

37. Dexheimer, P.J.; Cochella, L. MicroRNAs: From Mechanism to Organism. Front. Cell Dev. Biol. 2020, 8, 1-18. [CrossRef]

38. Zhou, K.; Zhang, T.; Fan, Y.D.; Serick; Du, G.; Wu, P.; Geng, D. MicroRNA-106b promotes pituitary tumor cell proliferation and invasion through PI3K/AKT signaling pathway by targeting PTEN. Tumor Biol. 2016, 37, 13469-13477. [CrossRef] [PubMed]

39. Zhang, N.; Lin, J.K.; Chen, J.; Liu, X.F.; Liu, J.L.; Luo, H.S.; Li, Y.Q.; Cui, S. MicroRNA 375 mediates the signaling pathway of corticotropin-releasing factor (CRF) regulating pro-opiomelanocortin (POMC) Expression by targeting Mitogen-activated protein Kinase 8. J. Biol. Chem. 2013, 288, 10361-10373. [CrossRef]

40. Grzywa, T.M.; Klicka, K.; Rak, B.; Mehlich, D.; Garbicz, F.; Zieliński, G.; Maksymowicz, M.; Sajjad, E.; Włodarski, P.K. Lineagedependent role of $\mathrm{miR}-410-3 \mathrm{p}$ as oncomiR in gonadotroph and corticotroph pituitary adenomas or tumor suppressor miR in somatotroph adenomas via MAPK, PTEN/AKT, and STAT3 signaling pathways. Endocrine 2019, 65, 646-655. [CrossRef] 